11

\title{
Прямое наблюдение квазилокализованной низкочастотной колебательной моды в спектре возбуждения флуоресценции одиночной примесной молекулы в полимерной матрице*
}

\author{
(C) А.О. Савостьянов ${ }^{1,2}$, И.Ю. Еремчев ${ }^{1,3}$, А.А. Горшелев ${ }^{1}$, С.В. Орлов ${ }^{1}$, А.С. Старухин ${ }^{4}$, А.В. Наумов ${ }^{1,3,5}$ \\ ${ }^{1}$ Институт спектроскопии РАН, \\ 108840 Троицк, Россия \\ ${ }^{2}$ Физический институт им. П.Н. Лебедева РАН, \\ 119991 Москва, Россия \\ ${ }^{3}$ Московский фризико-технический институт (государственный университет), \\ 141700 Долгопрудный, Россия \\ ${ }^{4}$ Институт фризики им. Б.И. Степанова НАН Беларуси, \\ 220072 Минск, Беларусь \\ ${ }^{5}$ Московский педагогический государственный университет, \\ 119991 Москва, Россия \\ e-mail: savostianov.a@isan.troitsk.ru
}

Поступила в редакцию 24.09.2018 г.

\begin{abstract}
Продемонстрирована возможность прямого детектирования квазилокализованного элементарного возбуждения фононного типа в полиизобутилене путем регистрации спектра возбуждения флуоресценции одиночной зондовой молекулы Mg-тетраазапорфирина в широком спектральном диапазоне при температуре $6 \mathrm{~K}$. Измерены параметры квазилокализованной низкочастотной колебательной моды (НЧМ): частота (энергия) в возбужденном электронном состоянии примесной молекулы $\left(\omega_{e}=13.94 \pm 0.21 \mathrm{~cm}^{-1}\right)$ и полуширина спектра НЧМ $\left(\gamma=3.82 \pm 0.66 \mathrm{~cm}^{-1}\right)$. Измеренное значение энергии НЧМ совпадает с положением максимума бозонного пика в спектре колебательных состояний полимера, что свидетельствует о применимости приближения слабой связи к рассмотренному случаю электрон-фононного взаимодействия молекулы органического красителя с локальным окружением в полимерной матрице.
\end{abstract}

DOI: $10.21883 /$ OS.2019.01.47053.286-18

\section{Введение}

Колебательная динамика неупорядоченных твердотельных сред (стекол) вообще и полимерных стекол в частности является предметом многочисленных исследований и дискуссий. Исследования разнообразных аморфных материалов с применением различных экспериментальных методов (в том числе с помощью неупругого нейтронного рассеяния [1,2], неупругого ядерного резонансного рассеяния [3], комбинационного рассеяния света [4-7], фотонного эха [8,9], терагерцовой спектроскопии поглощения [10]) продемонстрировали аномальный характер спектральной плотности колебательных состояний (ПКС), проявляющийся в появлении ярко выраженного асимметричного низкочастотного максимума, получившего название „бозонный пик“. Бозонный пик наблюдается для всех типов стекол, что, повидимому, означает, что его появление никак не связано с химической структурой определенного вещества, а определяется особенностями строения неупорядоченных сред на нанометровом масштабе. К настоящему времени существуют разнообразные подходы к объяснению возникновения бозонного пика, среди которых наиболь-

* XIII International Conference on Hole Burning, Single Molecule, and Related Spectroscopies: Science and Applications (HBSM-2018), August 6-12, 2018, Suzdal-Moscow, Russia. шее распространение получила концепция о существовании в стеклах помимо акустических фононов низкочастотных квазилокализованных колебательных мод (НЧМ) [11-14].

Среди экспериментальных методов исследования колебательной динамики стекол особое место занимает флуоресцентная спектромикроскопия одиночных молекул (СМОМ). В данном методе одиночные молекулы (ОМ), внедренные в исследуемую твердотельную матрицу (см. [15] и ссылки там), используются в качестве спектральных нанозондов. Чрезвычайная чувствительность бесфононных спектральных линий (БФЛ) хромофорных молекул при криогенных температурах к параметрам локального окружения позволяет исследовать колебательную динамику матрицы на масштабах от единиц до десятков нанометров, что по порядку величины соответствует рассчитанной области пространственной локализации НЧМ в стеклах [27,28].

Температурное уширение БФЛ ОМ, внедренных в полимерную матрицу, вызванное квадратичным электронфононным (ЭФ) взаимодействием, уже исследовалось ранее достаточно подробно [16-18]. Среди прочих поднимался вопрос о корректности применения приближения слабой связи, подразумевающего малость константы ЭФ взаимодействия и значительно упрощающего формулу, которая описывает зависимость ширины БФЛ от 
температуры и параметров НЧМ. Как было наглядно продемонстрировано в работе [26] для ансамбля молекул, а в работе [19] для ОМ, вышеупомянутое приближение достаточно точно описывает экспериментальные зависимости лишь для определенных частот НЧМ в узком диапазоне температур. В связи с вышесказанным становится весьма актуальным вопрос о получении параметров НЧМ непосредственно из анализа фононных крыльев (ФК), наблюдаемых в спектрах флуоресценции и спектрах возбуждения флуоресценции, в том числе и ОМ [20]. Извлеченные из эксперимента значения частоты НЧМ для основного и возбужденного электронных состояний примесной молекулы, а также значение полуширины спектра НЧМ позволяют точно вычислить величину константы квадратичного ЭФ взаимодействия, что далее может быть использовано для расчета температурной зависимости уширения БФЛ при любой силе ЭФ взаимодействия.

В настоящей работе приведены результаты измерений спектра возбуждения флуоресценции органической ОМ в полимерной матрице при криогенной температуре, которые позволили впервые наблюдать ФК и проявление одиночной НЧМ в его структуре.

\section{Экспериментальная часть}

В настоящей работе параметры НЧМ были получены напрямую из спектров возбуждения флуоресценции ОМ Mg-тетраазапорфирина (Mg-ТАП), внедренных в тонкие полимерные пленки полиизобутилена (ПИБ) производства Aldrich $\left(M_{w}=420000 \mathrm{~g} / \mathrm{mol}\right)$. Измерения проводились при $T=6 \mathrm{~K}$.

Mg-ТАП был синтезирован по модифицированной методике [24] и тщательно очищен хроматографически. Структура полученных соединений была установлена с помощью спектроскопии ЯМР, а чистота подтверждена методами ИК, абсорбционной и флуоресцентной спектроскопии. Полимерные пленки изготовлялись посредством центрифугирования: на вращающуюся тонкую стеклянную подложку наносилась капля растворенного в 2-метилтетрагидрофуране полимера с примесью молекул $\mathrm{Mg}$-ТАП. Остатки растворителя удалялись из образца с помощью вакуумирования. Концентрация примесных молекул подбиралась таким образом, чтобы в поле зрения микроскопа при перестройке частоты возбуждающего лазера всегда находилось небольшое (в пределах одного-двух десятков) число люминесцирующих ОМ.

Используемая экспериментальная установка была детально описана ранее в [25]. Измерения спектров возбуждения люминесценции осуществлялись путем синхронной перестройки частоты возбуждающего лазера и детектирования стоксовой компоненты люминесценции ОМ. В работе использовался перестраиваемый лазер на красителе (Р6Ж) Coherent CR-599 с эффективной шириной линии $10 \mathrm{GHz}\left(\sim 0.33 \mathrm{~cm}^{-1}\right)$. Перестройка частоты лазера производилась в широком (вплоть до
$4000 \mathrm{GHz}$ или $\sim 133 \mathrm{~cm}^{-1}$ ) спектральном диапазоне в области $575-595 \mathrm{~nm}$, что соответствует неоднородно уширенному контуру поглощения Mg-ТАП в ПИБ.

Для сбора и регистрации сигнала люминесценции от ОМ использовалась схема люминесцентного микроскопа (область фокусировки $\sim 30 \times 30 \mu \mathrm{m}$ ) с ПЗС-камерой (Andor iXon Ultra). C помощью полосового интерференционного фильтра (Semrock SR628/32) из сигнала, приходящего на камеру, выделялся спектральный диапазон 612-644 nm, в котором находится достаточно интенсивный пик вибронной полосы молекул $\mathrm{Mg}$-ТАП.

Светосильный микрообъектив (MellisGriot, 40X, $0.65 \mathrm{NA})$ и образец были зафиксированы на специально разработанном трансляционном модуле, который помещался на штоке в измерительной камере оптического терморегулируемого Не-4-криостата (RTI, Россия), позволяющего осушествлять измерения в широком температурном диапазоне вплоть до 1.6 К. Оптикомеханический транслятор обеспечивал прецизионное перемещение (в том числе при криогенных температурах) образца относительно микрообъектива в аксиальном направлении для размещения полимерной пленки в фокальной плоскости люминесцентного микроскопа.

\section{Результаты и их обсуждение}

В работе были измерены и детально исследованы более тридцати спектров возбуждения флуоресценции для электронных переходов в ОМ Mg-ТАП в тонких пленках ПИБ. Помимо узких БФЛ для части спектров были обнаружены сателлиты: широкие спектральные пики с малой (по сравнению с БФЛ) амплитудой, совершающие с соответствующими БФЛ синхронные во времени прыжки. Данные линии были сдвинуты относительно БФЛ в область больших энергий на величине $\sim 10-20 \mathrm{~cm}^{-1}$, что соответствует характерным значениям энергий НЧМ в полимерах и непосредственно в ПИБ [18]. Данные факты позволяют нам сделать предположение о том, что наблюдавшиеся сателлиты являются ФК в вибронной полосе исследованной ОМ. Необходимо отметить, что обнаруженные пики хорошо аппроксимируются функцией Лоренца, что отличает их от типичных ФК, наблюдавшихся во многих экспериментах с использованием стандартных методов селективной лазерной спектроскопии с ансамблевым усреднением. Для такого рода экспериментов характерны асимметричные формы ФК, содержащие хорошо различимые в спектре длинные „хвосты“, удаленные от БФЛ „на расстояния“ в несколько десятков обратных сантиметров. Как правило, появление столь широких ФК объяснялось линейным ЭФ взаимодействием электронного перехода примесной молекулы с широким спектром акустических фононов и полностью или частично локализованных колебательных возбуждений матрицы. В нашем же случае, по всей видимости, мы имеем дело с взаимодействием ОМ с одним ярко выраженным квазилокализованным 


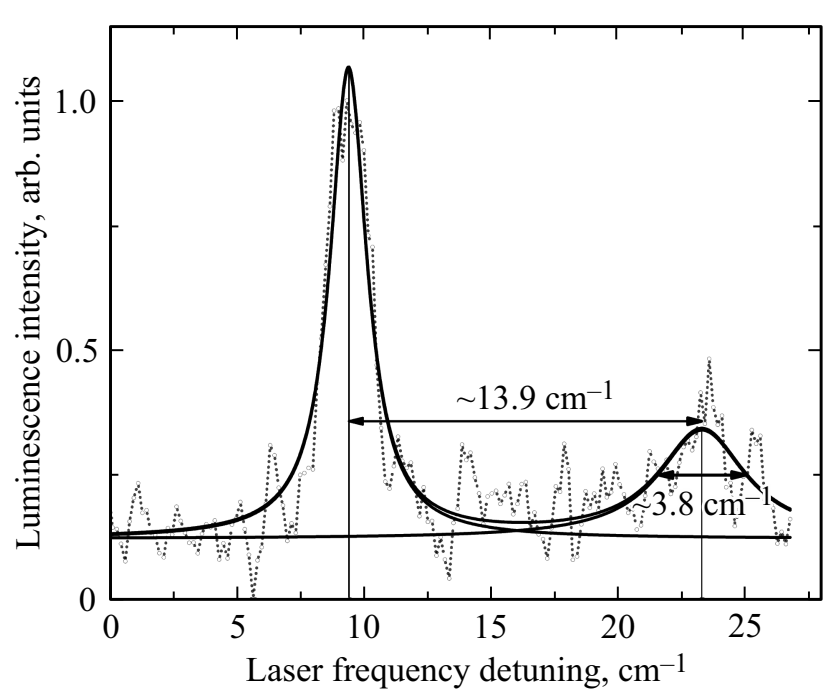

Рис. 1. Спектр возбуждения флуоресценции ОМ Mg-ТАП в тонкой пленке ПИБ. БФЛ и ФК аппроксимированы с помощью функции Лоренца. Полуширина спектра НЧМ $\gamma \approx 3.8 \mathrm{~cm}^{-1}$, частота $\omega_{e} \approx 13.9 \mathrm{~cm}^{-1}$.

колебанием [21]. При этом влияние всех остальных элементарных возбуждений фононного типа оказывается малым вследствие весьма низкой температуры $(6 \mathrm{~K})$.

На рис. 1 показан спектр возбуждения флуоресценции $\mathrm{OM} \mathrm{Mg-ТАП} \mathrm{в} \mathrm{ПИБ,} \mathrm{содержащий} \mathrm{БФЛ} \mathrm{и} \mathrm{ФК.}$ Измеренный спектр был аппроксимирован с помощью двух функций Лоренца. Полученные в результате аппроксимации значения полуширины БФЛ и ФК составили $\Gamma_{Z P L}=1.43 \pm 0.09 \mathrm{~cm}^{-1}$ и $\gamma=3.82 \pm 0.66 \mathrm{~cm}^{-1}$ соответственно (значения получены с учетом вклада от аппаратной функции лазера). Частота НЧМ в возбужденном электронном состоянии (сдвиг максимума ФК относительно БФЛ) составила значение $\omega_{e}=13.94 \pm 0.21 \mathrm{~cm}^{-1}$. Необходимо отметить, что измеренное значение $\Gamma_{\mathrm{ZPL}}$ значительно превосходит (в $\sim 5-50$ раз) ширины БФЛ, наблюдаемых ранее в ПИБ для ОМ тетра-трет-бутилтеррилена (ТБТ) и дибензоантантрена (ДБАТТ) [18]. Есть все основания предположить, что столь значительные отличия связаны с большим вкладом быстрой (по сравнению с характерным временем измерения) спектральной диффузии в уширение БФЛ.

Полученное в результате аппроксимации значение частоты НЧМ хорошо согласуется с данными, полученными ранее различными экспериментальными методами для ПИБ. Так, на рис. 2 показаны: энергетический спектр НЧМ, полученный из анализа измеренных зависимостей температурного уширения БФЛ (с использованием приближения слабой связи) для 155 молекул ДБАТТ и 281 молекулы ТБТ в ПИБ [18]; значение „эффективной“ фононной моды, измеренное с помощью метода фотонного эха для системы ТБТ/ПИБ [8]; бозонный пик, измеренный методом неупругого нейтронного рассеяния для чистого ПИБ без примесей [2]. Для удобства ПКС приведена в нормированном виде $\left(g(\omega) / \omega^{2}\right)$, чтобы наглядно продемонстрировать вклад в ПКС акустических фононов, чей спектр описывается функцией Дебая (пунктирная линия на рисунке).

Как хорошо видно из рис. 2, измеренный в эксперименте спектр одиночной НЧМ в полной мере соответствует диапазону энергий, в котором расположен бозонный пик для исследованного материала ПИБ. Найденное нами значение $\omega_{e}$ соответствует положению максимума бозонного пика, измеренного как для чистого, так и для примесного ПИБ. Этот результат свидетельствует о том, что внедренная примесная ОМ не оказывают значительного влияния на локальную колебательную динамику (как это было показано ранее в [18]).

Однородное уширение БФЛ ОМ $\Gamma_{\text {Phon, }}$ вызванное квадратичным ЭФ взаимодействием, может быть описано в общем виде с помощью общей формулы, полученной Осадько [21] и позднее подтвержденной Скиннером и Хсу $[22,23]$ :

$$
\Gamma_{\text {Phon }}=\int_{0}^{\infty} \frac{d \omega}{2 \pi} \ln \left[1+4 n(\omega)(n(\omega)+1) W^{2} \Gamma^{e}(\omega) \Gamma^{g}(\omega)\right],
$$

где $n(\omega)-$ бозе-фактор, $W-$ константа квадратичного ЭФ взаимодействия, $\Gamma^{g}$ и $\Gamma^{e}-$ спектральные ПКС (фононов) соответственно для основного и возбужденного состояний примесных молекул. В случае взаимодействия электронного перехода примесной ОМ с квазилокализованным колебанием функция $\Gamma^{g}(\omega)$ обычно описывается функцией Лоренца, при этом значение $W$ определяется

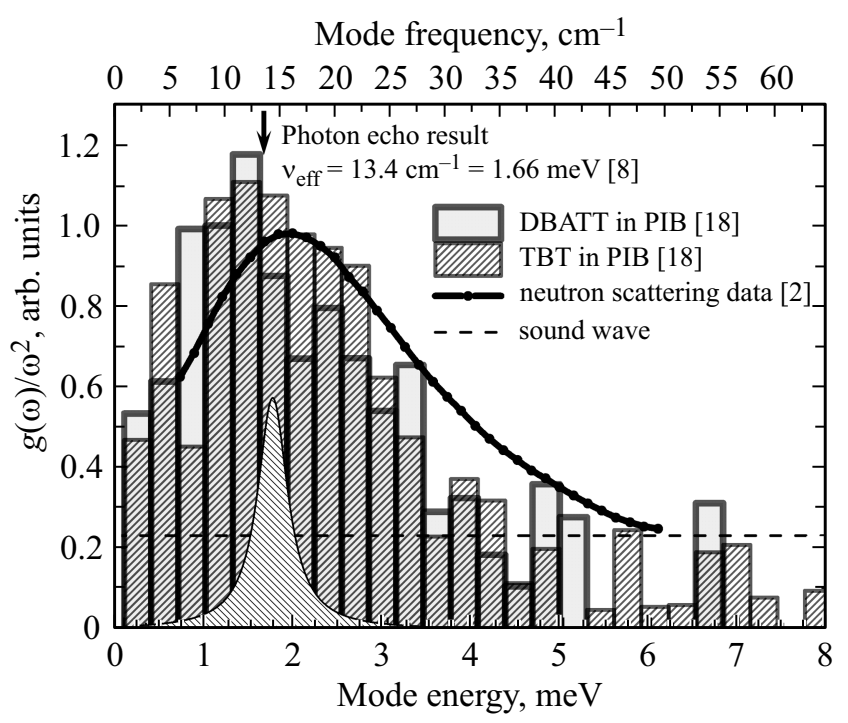

Рис. 2. Энергетический спектр НЧМ в ПИБ в нормированном виде $g(\omega) / \omega^{2}$ получен в различных экспериментах (подробности в тексте). Лоренцев профиль соответствует параметрам одиночной НЧМ, полученным из анализа спектров возбуждения флуоресценции $\mathrm{Mg}$-ТАП в ПИБ. Штрихованная прямая плотность колебательных мод в приближении Дебая. 
только параметрами НЧМ:

$$
W=\frac{\omega_{e}^{2}-\omega_{g}^{2}}{2},
$$

где $\omega_{g}$ и $\omega_{e}-$ частоты НЧМ в основном и возбужденном состояниях молекулы.

Универсальность выражения (1) состоит в том, что оно справедливо при любой силе ЭФ взаимодействия и любых температурах в отличие от широко используемого приближения слабой связи, при котором формула (1) значительно упрощается:

$$
\Gamma_{\text {Phon }}=B \frac{\exp (-\hbar \omega / k T)}{[1-\exp (-\hbar \omega / k T)]^{2}},
$$

где $B=W^{2} / \gamma \omega^{2}, \gamma-$ ширина спектра НЧМ. Критерий применимости такого приближения $-b \ll 1$, где $b-$ безразмерная константа связи, равная $b=W / \gamma \omega$. Частота $\omega=\omega_{g} \approx \omega_{e}$.

В общем случае частоты $\omega_{g}$ и $\omega_{e}$ заметно отличаются. Для того чтобы однозначно вычислить однородное уширение БФЛ по формуле (1), необходимо знать частоты НЧМ и для основного, и для возбужденного состояний примесной молекулы, что возможно при измерении сопряженных спектров флуоресценции и возбуждения флуоресценции примесной ОМ. Подобный эксперимент ранее был проведен для ансамбля молекул 3,4,5,6-дибензпирена в н-октане [26]. Было показано, что даже в случае значительного ансамблевого усреднения, существует возможность нахождения всего набора параметров $\omega_{g}, \omega_{e}, \gamma$, что далее позволяет рассчитать температурное уширение БФЛ, вызванное ЭФ взаимодействием.

В нашем случае показано, что такие параметры могут быть напрямую измерены и для примесной ОМ, взаимодействующей с локальным окружением в полимерной матрице. При этом доминирующим оказывается влияние одиночной квазилокализованной НЧМ. Следует также отметить, что измеренное значение $\omega_{e}$ позволяет сделать вывод о справедливости приближения слабой связи для рассмотренного случая, поскольку $\omega_{e}$ соответствует положению максимума бозонного пика, измеренного для чистого ПИБ, т.е. $\omega_{e} \approx \omega_{g}$.

Таким образом, в работе продемонстрирована возможность прямого детектирования квазилокализованной колебательной НЧМ в спектре возбуждения флуоресценции ОМ Mg-ТАП в прозрачной тонкой пленке аморфного полиизобутилена с использованием техники криогенной флуоресцентной спектромикроскопии. Спектр НЧМ удовлетворительно описывается функцией Лоренца, что дает возможность определить параметры этого элементарного возбуждения: частоту (энергию) НЧМ для возбужденного электронного состояния примесной молекулы $\left(\omega_{e}=13.94 \pm 0.21 \mathrm{~cm}^{-1}\right)$, а также ширину спектра НЧМ $\left(\gamma=3.82 \pm 0.66 \mathrm{~cm}^{-1}\right)$. Измеренные параметры могут быть использованы в расчетах уширения БФЛ примесной молекулы вследствие квадратичного ЭФ взаимодействия. Полученное значение частоты НЧМ $\omega_{e}$ соответствует положению максимума в энергетическом спектре (плотности) колебательных состояний в низкотемпературном ПИБ, независимо измеренном ранее с использованием других методов. Этот факт свидетельствует о применимости приближения слабой связи ЭФ взаимодействия к рассмотренному случаю примесной OM Mg-ТАП, взаимодействующей с локальным окружением матрицы ПИБ.

Работа выполнена при поддержке программы Президиума РАН I.7 „Актуальные проблемы фотоники, зондирование неоднородных сред и материалов“. Часть работы (А.С.С) выполнена при поддержке программы „Горизонт-2020“. Работа российских авторов проводилась в рамках выполнения Государственного задания Института спектроскопии РАН.

\section{Список литературы}

[1] Buchenau U., Prager M., Nücker N., Dianoux A.J., Ahmad N., Phillips W.A. // Phys. Rev. B. 1986. V. 34. P. 5665.

[2] Inoue K., Kanaya T., Ikeda S., Kaji K., Shibata K., Misawa M., Kiyanagi Y. // J. Chem. Phys. 1991. V. 95. P. 5332.

[3] Sette F., Krisch M.H., Masciovecchio C., Ruocco G., Monaco G. // Science. 1998. V. 280. P. 1550.

[4] Shuker R., Gammo R. W. // Phys. Rev. Lett. 1970. V. 25. P. 222.

[5] Brodin A., Börjesson L., Engberg D., Torell L.M., Sokolov A.P. // Phys. Rev. B. 1996. V. 53. P. 11511.

[6] Wiedersich J., Surovtsev N.V., Rössler E. // J. Chem. Phys. 2000. V. 113. P. 1143.

[7] Hehlen B., Courtens E., Vacher R., Yamanaka A., Kataoka M., Inoue K. // Phys. Rev. Lett. 2000. V. 84. P. 5355.

[8] Vainer Y.G., Kol'chenko M.A., Naumov A.V., Personov R.I., Zilker S.J. // J. Lumin. 2000. V. 86. P. 265.

[9] Vainer Y.G., Kol'chenko M.A., Naumov A.V., Personov R.I., Zilker S.J., Haarer D. // J. Chem. Phys. 2002. V. 116. P. 8959.

[10] Naftaly M., Miles R.E. // J. Non-Cryst. Solids. 2005. V. 351. P. 3341.

[11] Graebner J.E., Golding B., Allen L.C. // Phys. Rev. B. 1986. V. 34. P. 5696.

[12] Duval E., Boukenter A., Achibat T. // J. Phys.: Condens. Matter. 1990. V. 2. P. 10227.

[13] Laird B.B., Schober H.R. // Phys. Rev. Lett. 1991. V. 66. P. 636.

[14] Buchenau U., Galperin Yu.M., Gurevich V.L., Schober H.R. // Phys. Rev. B. 1991. V. 43. P. 5039.

[15] Наумов А.В. // УФН. 2013. Т. 183. Вып. 6. С. 633-652.

[16] Naumov A.V., Vainer Y.G., Kador L. // Phys. Rev. Lett. 2007. V. 98. P. 145501.

[17] Vainer Y.G., Naumov A.V., Kador L. // Phys. Rev. B. 2008. V. 77. P. 224202.

[18] Eremchev I.Y., Naumov A.V., Vainer Y.G., Kador L. // J. Chem. Phys. 2009. V. 130. P. 184507.

[19] Naumov A.V., Vainer Y.G., Kador L. // Phys. Rev. B. 2009. V. 79. P. 132201.

[20] Савостьянов А.О., Ерёмчев И.Ю., Горшелев А.А., Наумов А.В., Старухин А.С. // Письма в ЖЭТФ. 2018. № 107. C. 426 . 
[21] Осадько И.С. Квантовая динамика молекул, взаимодействующих с фотонами, фононами и туннельными системами. М.: Физматлит, 2017. 368 с.

[22] Hsu D., Skinner J.L. // J. Chem. Phys. 1984. V. 81. P. 5471.

[23] Hsu D., Skinner J.L. // J. Chem. Phys. 1985. V. 83. P. 2097.

[24] Linstead R.P., Whalley M. // J. Chem. Soc. (Resumed). 1952. P. 4839.

[25] Naumov A., Eremchev I.Y., Gorshelev A.A. // Eur. Phys. J. D. 2014. V. 68. P. 348.

[26] Коротаев О.Н., Калитеевский М.Ю. // ЖЭТФ. 1980. № 79. C. 439.

[27] Schober H.R., Oligschleger C. // Phys.Rev. B. 1996. V. 53. P. 11469.

[28] Schober H.R. // J. Non-Cryst. Sol. 2002. V. 307. P. 40. 